\title{
$5 \mathrm{~Hz}$ Repetitive Transcranial Magnetic Stimulation for Posttraumatic Stress Disorder Comorbid with Major Depressive Disorder
}

\author{
Linda L. Carpenter, MD ${ }^{a},{ }^{\star}$, Christine Conelea, PhD ${ }^{c, b}$, Audrey R. Tyrka, MD PhD ${ }^{a}$, Emma S. \\ Welch, MSa, Benjamin D. Greenberg, MD PhD ${ }^{a, b}$, Lawrence H. Price, MDa, Matthew \\ Niedzwiecki, MDa ${ }^{a}$ Agustin G. Yip, MD PhDa , Jennifer Barnes, MDa ${ }^{a}$, and Noah S. Philip, \\ $\mathrm{MD}^{\mathrm{a}, \mathrm{b}}$ \\ aButler Hospital, Department of Psychiatry and Human Behavior, Alpert Medical School of Brown \\ University \\ ${ }^{\mathrm{b}}$ Center for Neurorestoration and Neurotechnology, Providence VA Medical Center, Providence RI \\ 'Bradley Hospital, Department of Psychiatry and Human Behavior, Alpert Medical School of \\ Brown University
}

\section{Abstract}

Background: Standard clinical protocols for repetitive transcranial magnetic stimulation (rTMS) for major depressive disorder (MDD) apply $10 \mathrm{~Hz}$ pulses over left prefrontal cortex, yet little is known about the effects of rTMS in more diagnostically complex depressed patients.

Objective/Hypothesis: Posttraumatic stress disorder (PTSD) is commonly comorbid with MDD, and while rTMS has been shown to alleviate PTSD symptoms in preliminary studies, ideal parameters remain unclear. We conducted a prospective, open-label study of $5 \mathrm{~Hz}$ rTMS for patients with comorbid PTSD+MDD and hypothesized stimulation would reduce symptoms of both disorders.

Methods: Outpatients (N=40) with PTSD+MDD and at least moderate global severity were enrolled. $5 \mathrm{~Hz}$ rTMS included up to 40 daily sessions followed by a 5 -session taper. Symptoms were measured using the PTSD Checklist (PCL-5) and Inventory of Depressive Symptomatology, Self-Report (IDS-SR). Baseline-to-endpoint changes were analyzed.

Results: The intent-to-treat population included 35 participants. Stimulation significantly reduced PTSD symptoms (PCL-5 baseline mean \pm SD score 52.2 \pm 13.1 versus endpoint 34.0 \pm 21.6 ; $\mathrm{p}<.001) ; 23$ patients (48.6\%) met a pre-defined categorical PTSD response criteria. MDD symptoms also improved significantly (IDS-SR, baseline $47.8 \pm 11.9$ to endpoint $30.9 \pm 18.9 ; \mathrm{p}<$. $001) ; 15$ patients (42.9\%) demonstrated categorical response and 12 (34.3\%) remitted. PTSD and MDD symptom change was highly correlated $(\mathrm{r}=.91, \mathrm{p}<.001)$.

*Address correspondence to Linda L. Carpenter, M.D., Butler Hospital, 345 Blackstone Blvd, Providence RI 02906; 401.455.6349; Linda_Carpenter_MD@Brown.edu.

Drs. Conelea, Yip, and Barnes completed work associated with the current study at the listed institutions but are now affiliated with the University of Minnesota (Conelea) and McLean Hospital/Harvard Medical School (Yip and Barnes). 
Limitations: Unblinded single-arm study, with modest sample size.

Conclusion: Significant and clinically meaningful reductions in both MDD and PTSD symptoms were observed following stimulation. The preliminary efficacy of $5 \mathrm{~Hz}$ rTMS for both symptom domains in patients with comorbid disorders supports future controlled studies.

(5 Hz Repetitive Transcranial Magnetic Stimulation for Posttraumatic Stress Disorder Comorbid With Major Depressive Disorder; Clinicaltrials.gov; NCT02273063)

\section{Keywords}

repetitive transcranial magnetic stimulation; posttraumatic stress disorder; major depressive disorder; $5 \mathrm{~Hz}$

\section{INTRODUCTION}

Repetitive transcranial magnetic stimulation (rTMS) therapy has emerged as an important standard-of-care treatment for adults with major depressive disorder (MDD) that do not benefit from standard antidepressant treatments. Due to concerns about comorbid psychiatric conditions introducing disease heterogeneity into study samples (O'Reardon et al., 2007; George et al., 2010; Levkovitz et al., 2015) regulatory MDD rTMS trials systematically excluded adults who were concurrently experiencing threshold symptoms of comorbid anxiety, obsessive-compulsive and/or posttraumatic stress disorder (PTSD). In real-life practice, however, clinicians are faced with the challenge of treating patients who are more diagnostically complex than those who meet stringent research clinical trial eligibility criteria.

PTSD is a common psychiatric disorder that can result in significant symptoms and psychosocial dysfunction. It is estimated that approximately $7 \%$ of the U.S. population experiences PTSD over the course of their lifetime (Kessler et al., 2005), and 50-70\% of Veterans are diagnosed with PTSD (Hoge et al., 2004). Exposure to severe traumatic events is common among civilians living in urban areas, and PTSD occurs in one third of those exposed (Alim et al., 2008). PTSD is associated with diminished physical health and unemployment (Thomas et al., 2010), making it an important public health problem. Furthermore, PTSD and MDD are highly comorbid; up to 50\% of patients with PTSD are also diagnosed with MDD (Rytwinkski et al., 2013; Flory and Yehuda, 2015). In both disorders, a substantial number of patients remain symptomatic despite standard treatments (Trivedi et al., 2006; Rush et al., 2006; Watts et al., 2013). Patients with comorbid MDD and PTSD symptoms have poorer treatment outcomes for both pharmacotherapy and psychotherapy (Holtzheimer et al., 2005; Campbell et al., 2007) and as such are well represented among those with treatment-resistant major depression referred for rTMS therapy.

A handful of small pilot studies (see review (Karsen et al., 2014)) suggest rTMS therapy holds potential for improving PTSD symptoms. However, while rTMS has been developed as an effective treatment for depression over the past decade, less attention has been focused on determining its use for PTSD. Optimal rTMS stimulation parameters have not been identified for treatment when PTSD is a comorbid disorder. Boggio et al. (2010) found that 
10 sessions of rTMS delivered to PTSD patients $(\mathrm{N}=30)$ at a relatively high frequency (20 $\mathrm{Hz}$ ) on either the left or right prefrontal cortex (PFC) was associated with significant clinical improvement compared to sham. Left-sided stimulation appeared to specifically benefit mood symptoms, whereas right-sided treatment was associated with greater improvement in anxiety symptoms. Watts et al. (2012) reported significant and persistent improvement in PTSD symptoms alongside significant mood benefits following delivery of 10 rTMS sessions to the right PFC at a low frequency $(1 \mathrm{~Hz})$ in a PTSD sample $(\mathrm{n}=20)$.

Successful use of both higher $(20 \mathrm{~Hz})$ and lower $(1 \mathrm{~Hz})$ pulse frequencies in these pilot studies suggest that magnetic fields delivered in a broad range of pulse frequencies induce physiologic effects on neuronal function. For MDD, a compelling evidence base exists for $10 \mathrm{~Hz}$ rTMS (over left PFC) using a figure- 8 coil, or $18 \mathrm{~Hz}$ stimulation (over left $>$ right bilateral PFC) with an H-coil (McClintock et al., 2017). A growing body of work has also demonstrated that $1 \mathrm{~Hz}$ rTMS to PFC on the right side produces significant antidepressant effects (Berlim et al., 2013), as well as prominent anxiolytic effects in patients with anxious depression (Rossini et al., 2010).

Owing to the sheer number of stimulation parameters contributing to the overall "dose" of rTMS and the need to manipulate only one in each clinical trial, relatively little has been done to systematically test efficacy of stimulation between 1 and $10 \mathrm{~Hz}$. Several studies have employed a stimulation frequency of $5 \mathrm{~Hz}$ (left PFC) and reported outcomes equivalent to those achieved with $10 \mathrm{~Hz}$ for treating depression in bipolar patients (George et al., 2000; Nahas et al., 2003; Rumi et al., 2005; Su, et al., 2005; Avery, et al., 2008). Fitzgerald et al. (2009) randomized MDD patients (nonresponders to right-sided $1 \mathrm{~Hz}$ rTMS) to receive either $5 \mathrm{~Hz}$ or $10 \mathrm{~Hz}$ stimulation on the left side, and found equivalent antidepressant efficacy. $5 \mathrm{~Hz}$ stimulation using an $\mathrm{H}$-coil produced significant improvement in social anxiety and social relatedness when compared with sham in an autism sample (Enticott et al., 2014). An open-label study in patients with comorbid MDD+PTSD (Rosenberg et al., 2002) demonstrated modest improvements in both mood and PTSD symptoms when $1 \mathrm{~Hz}$ and $5 \mathrm{~Hz}$ stimulation were delivered over left PFC, but the rTMS treatment protocol included only 600 pulses/day and a total of only 10 sessions, which would be considered a very low or inadequate "dose" of rTMS by current standards.

Our group has systematically administered $5 \mathrm{~Hz}$ rTMS therapy to MDD patients in our clinics to address high levels of comorbid anxiety or activation emerging during standard 10 $\mathrm{Hz}$ therapy, which can be particularly problematic when rTMS is given as an adjunct to ongoing monoaminergic antidepressants or other stimulating medications (Philip et al., 2015). MDD efficacy outcomes for patients receiving $5 \mathrm{~Hz}$ rTMS (to left PFC) were equivalent to those getting $10 \mathrm{~Hz}$, and the slower pulse frequency was better tolerated. We also examined acceptability and safety of $5 \mathrm{~Hz}$ rTMS in a case series $(\mathrm{n}=10)$ and reported reductions in PTSD and MDD symptoms, along with excellent tolerability (Philip et al., 2016).

This literature and our prior experience laid the groundwork for a prospective open-label study of rTMS delivered at $5 \mathrm{~Hz}$ over the left (dorsolateral) PFC in patients with comorbid PTSD and MDD, designed to both characterize efficacy and safety of the protocol. We 
hypothesized that $5 \mathrm{~Hz}$ stimulation over left PFC would significantly reduce both PTSD and MDD symptoms with a safety and tolerability profile comparable to prior rTMS studies.

\section{METHODS}

\section{Study overview}

This study was a prospective, open-label trial of rTMS delivered to outpatients who were symptomatic despite ongoing naturalistic pharmacotherapy for PTSD and MDD. The study occurred at two collaborating sites associated with the Alpert Medical School of Brown University: Butler Hospital (BH) and the Providence Veteran's Administration Medical Center (PVAMC). The institutional review boards (IRBs) at both sites approved the study, and all participants provided written informed consent for participation in the trial (ClinicalTrials.gov NCT02273063).

Eligibility was determined during an in-person screening visit. Eligible participants returned within two weeks to complete baseline assessments and begin rTMS. The rTMS treatment course allowed up to 40 sessions, delivered once per day, with the first 35 delivered on weekdays over 7 weeks and the final 5 on a taper schedule over 3 weeks. The maximum of 40 treatments for this acute series was informed by prior research suggesting that longer acute phase may be associated with superior clinical outcomes (Avery et al., 2008). Patients who achieved remission (operationally defined as 2 consecutive weeks of scores consistent with remission using measures described below) prior to treatment 35 could be tapered.

\section{Participants}

Participants were adults aged 18-75 years who met DSM-IV criteria for both PTSD (acute or chronic) and MDD (nonpsychotic, recurrent, or chronic) at the time of the screening and baseline visits. Clinicians with experience treating both disorders (NSP, LLC) verified the diagnoses. Either diagnosis could be considered "primary," but both disorders must have separately met an overall illness severity rating of at least "moderately ill" on the Clinical Global Impressions-Severity Scale (CGI-S (Guy, 1976)). Participants lacked response to, or demonstrated intolerance to, at least one antidepressant medication trial as part of definitive and adequate treatment in the current depressive episode; were on stable psychotropic regimens for at least six weeks prior to enrollment; could independently read and understand study measures/instructions; and females with child bearing potential agreed to use acceptable birth control methods. Stable, ongoing psychotherapy was permitted during the study. These participants were unique from our prior reports of $5 \mathrm{~Hz}$ rTMS for depression (Philip et al., 2015) and PTSD (Philip et al., 2016).

Candidates were excluded for: past rTMS treatment; breastfeeding, pregnancy or plans to become pregnant within the next three months; lifetime history of significant head injury (i.e., resulting in loss of consciousness greater than 10 minutes or documented evidence of brain injury); current/past significant neurological disorder (e.g., seizure disorder, brain tumors, stroke, cerebral aneurysm); current psychotic, bipolar I, alcohol and/or substance dependence or abuse disorders; active suicidal intent or plan, or any other contraindication to study procedures. 


\section{Assessments}

Primary outcomes for this study were change in PTSD and MDD symptom severity from baseline to post-treatment. The PTSD Checklist for DSM-5 (PCL-5 (Weathers et al., 2013) was used to measure PTSD symptoms, and MDD severity was measured using the Inventory of Depressive Symptomology-Self Report (IDS-SR (Rush et al., 1996)). Both scales were administered at baseline, biweekly during the first 4 weeks, weekly during the remainder of treatment, and within 72 hours after the final treatment session. Scores on the PCL-5 were examined for clinically meaningful improvement ( $\geq 10$ point reduction from baseline, per Weathers et al. (2013)) and categorical response (operationally defined as a score of $₫ 3$ at post-treatment together with clinically meaningful improvement). Clinical response on the IDS-SR was defined as a $250 \%$ reduction from baseline score, and remission as IDS-SR score of $\unlhd 4$ at post-treatment.

Secondary outcome measures administered at baseline and post-treatment assessed change in overall clinical state as determined by clinician and patient ratings of global illness severity (CGI-S and the equivalent patient self-rated version, PGI-S) and baseline-toendpoint global improvement (CGI-I and PGI-I). Additional measures of pre- and post-TMS symptom severity included the Patient Health Questionnaire (PHQ-9 (Kroenke et al., 2001)), the 42-item Depression Anxiety Stress Scale (DASS (Lovibond and Lovibond, 1995)), and the Perceived Stress Scale (PSS (Cohen et al., 1983)).

Baseline measures of childhood/adolescent exposure to abuse and neglect were assessed with the 28-item Childhood Trauma Questionnaire (CTQ (Bernstein et al., 2003)). Additional lifetime traumatic event detail was captured with the Life Events Checklist (LEC (Weathers et al., 2013)). LEC items endorsed as "happened to me" or "witnessed" were counted.

Treatment-emergent side effects, captured by spontaneous report and in response to specific daily queries made by TMS staff during the course of treatment, were categorized as serious or nonserious, and any interventions taken to address emergent adverse effects were recorded.

\section{TMS device and treatment parameters}

Prior to the first treatment session, the motor threshold (MT) was obtained in order to determine treatment intensity using the NeuroStar TMS Therapy System (Neuronetics, Inc., Malvern, PA). The same device was used for subsequent rTMS treatments delivered over the left PFC, determined by the standard F3 location using the international EEG 10/20 system measurements. Stimulation parameters were as follows: frequency $=5 \mathrm{~Hz}, 5 \mathrm{sec}$ stimulation train, 14 sec inter-train interval, 3000 pulses per session, intensity $120 \%$ MT. Per standard clinical practice, total pulses per session was increased to 4000 (with the inter-train interval shortened by $1 \mathrm{sec}$ ) for the remainder of the treatment course when participants did not have at least 30\% improvement from baseline scores at treatment 15. 


\section{Statistical Analyses}

Data were analyzed in an intent-to-treat (ITT) fashion, such that any participant who signed informed consent and received at least one rTMS session were included in the ITT sample, using the last observation carried forward (LOCF) method. Descriptive statistics were used to examine baseline sample characteristics. Paired sample-t tests characterized outcomes post-treatment on continuous measures (PCL-5, IDS-SR, PHQ-9, DASS, PSS, CGI-S, PGIS). Effect sizes were calculated for primary outcome measures PCL-5 and IDS-SR using Cohen's $d_{a V}$ (Lakens, 2013). Pearson correlations evaluated the relationship between PTSD and MDD symptom change following rTMS. We also examined potential variables of interest as predictors of clinical response, including depressive and PTSD symptom severity (total and subscale values), and baseline values of stress and anxiety on the DSS. Analyses were conducted in SPSS version 22 (IBM, Armonk, NY).

\section{RESULTS}

\section{Demographic and clinical characteristics}

Forty participants signed informed consent, and 35 comprised the ITT population. Reasons for dropout prior to starting rTMS included failure to meet eligibility criteria upon screening $(\mathrm{n}=2)$ and withdrawal of consent/changed mind $(\mathrm{n}=3)$. Demographic and clinical characteristics $(n=35)$ are presented in Table 1 . The mean \pm SD age was $51.6 \pm 10.3$. Sixty percent had a past history of inpatient psychiatric hospitalization, and $48.6 \%$ had prior intensive outpatient or partial hospitalization. Reflecting the significant disability associated with comorbid PTSD and MDD, $68.5 \%(\mathrm{n}=24)$ were either unemployed or on disability. Trauma exposure included a broad range of LEC trauma types and $82.9 \%$ of participants reported a history of at least "moderate" childhood maltreatment on one or more CTQ subscales. No patients with Bipolar II were enrolled in the study.

Mean $( \pm \mathrm{SD})$ number of treatment sessions was $32.9 \pm 9.3$, range 5-40. Thirty $(86 \%)$ patients completed final assessments after their last treatment session. The remaining five did not complete due to treatment-emergent adverse events or withdrawal of consent/lost to followup (see Safety Outcomes, below).

\section{Clinical outcomes}

Clinical outcomes are summarized in Table 2. Results show significant improvement in primary outcomes of both PTSD and MDD severity. PCL-5 scores significantly decreased from baseline (mean $\pm \mathrm{SD}, 52.2 \pm 13.1$ ) to post-treatment (34.0 $\pm 21.6 ; t=5.6, p<.001)$, with a mean $35.5 \%$ change $( \pm 38.0 \%$; range -50.0 to $90.9 \%)$ and a large observed effect size $\left(d_{a v}=\right.$ 1.04). IDS-SR scores also significantly decreased from baseline (47.8 \pm 11.9$)$ to posttreatment $(30.9 \pm 18.9 ; t=6.6, p<.001)$. Mean IDS-SR percent change was $37.6 \%( \pm 32.3 \%$, range -26.5 to $86.0 \%)$, and the effect size was large $\left(d_{a v}=1.09\right)$. There was a trend-level site difference in percent change on IDS-SR ( $p<.10)$, but outcomes were equivalent across sites on other measures. Percent change scores on the IDS-SR and PCL-5 were highly correlated $(\mathrm{r}=.92, \mathrm{p}<.001)$. 
Fifteen patients (42.9\%) met the IDS-SR response criterion and 12 (34.3\%) met remission criterion. Twenty-three patients (65.7\%) experienced clinically meaningful improvement (PCL-5 reduction of $\geq 10$ points), and 17 (48.6\%) met operational criteria for response. Fourteen $(40.0 \%)$ patients met response criteria on both MDD and PTSD measures.

Results also showed significant improvements on all secondary outcome measures (see Table 2). Clinician and patient ratings on the CGI-S/PGI-S scores were significantly better at study endpoint, and improvement ratings (CGI-I) indicated nearly half of patients were either "much improved" (11.4\%) or "very much improved" (34.3\%). One participant (2.9\%) was rated "minimally worse."

Post-hoc exploratory analyses were conducted to evaluate whether baseline severity of MDD or PTSD symptoms was related to "global response" status, defined as meeting response criteria for both IDS-SR and PCL; Table 3. A significant negative correlation was found between baseline depression severity and global response $\left(r_{p b}=-.38, p=.01\right)$, suggesting patients with more severe depression symptoms at baseline were less likely to have robust response in both diagnostic domains. Global response status was also negatively correlated with baseline PCL-5 Cluster C Avoidance score $\left(r_{p b}=-.42, p=.01\right)$, i.e., greater levels of PTSD-related avoidance were associated with lower likelihood of responding. Global response status was not significantly related to other measures of baseline PTSD severity or to baseline anxiety or stress ratings. All other predictors of response were nonsignificant (all $\mathrm{p}>$.1).

\section{Safety outcomes}

Four (11.4\%) patients experienced serious adverse events; three required hospitalization for worsening symptoms with suicidality, and one for suicidality and substance abuse. One experienced exacerbation of migraine and stopped after treatment \#31 due to headaches and lack of clinical response. Fourteen (40\%) experienced at least mild activation (defined as emergence or worsening of insomnia, irritability, anxiety, or agitation) during the course of TMS; all but one of these was taking stimulants or bupropion. Benzodiazepines were added to address activation in two cases, and dose reduction or discontinuation of a stimulating medication (bupropion or stimulants) was instituted in the others. Following this observation, Fisher's Exact tests were used to explore the relationship between stimulant/ bupropion use and activation, and we found a significant relationship between TMSemergent activation and ongoing concurrent stimulant/bupropion-treatment $(\mathrm{p}<.001)$.

\section{DISCUSSION}

This is the first report describing potential efficacy of $5 \mathrm{~Hz}$ rTMS to target symptoms of comorbid PTSD and MDD. Notwithstanding limitations of an unblinded prospective trial design, our results indicate $5 \mathrm{~Hz}$ rTMS delivered to left dorsolateral PFC (as adjunct to ongoing ineffective pharmacotherapy) is generally safe and significantly reduces symptoms of both disorders. Symptom improvement in both MDD and PTSD domains was observed, and $40 \%$ of the sample met criteria for categorical response on both the MDD and PTSD severity scales. General safety of $5 \mathrm{~Hz}$ stimulation was confirmed, though several patients taking concurrent medications with stimulating effects on the central nervous system (CNS) 
required dose reduction or medication discontinuation so they could continue with the 5 days/week rTMS schedule.

The presence of a comorbid anxiety disorder was previously reported to predict inferior symptom outcomes in a large randomized trial of patients with primary MDD (Lisanby et al., 2009). The patients in our sample epitomized a cohort that would be excluded from regulatory trials by virtue of their active diagnostic and clinical comorbidity, reliance on complex pharmacotherapy regimens despite minimal substantive benefit, past treatment history (e.g., $60 \%$ previously treated on an inpatient basis), and history of significant early life trauma. Nevertheless, we observed large effect sizes with $5 \mathrm{~Hz}$ rTMS, comparable to other studies of rTMS for PTSD (Clark et al., 2015) and for pharmacoresistant MDD (O'Reardon et al., 2007). We observed a 43\% antidepressant response rate and 34\% depressive episode remission rate, both comparable to outcomes for primary MDD patients in an industry-sponsored prospective open-label MDD trial that used restrictive inclusion/ exclusion criteria (Avery et al., 2008). While preliminary in nature, our results suggest that rTMS can be successfully used in patients with an otherwise poor prognosis and for whom the treatment may represent a desirable alternative option to electroconvulsive therapy.

There were no seizures and only one patient experienced headaches of sufficient intensity and duration to prompt early discontinuation after six weeks of daily sessions. Three of the 4 cases where suicidal ideation prompted transfer to inpatient care occurred near the end of the acute course or during the taper phase, as patients approached the maximum allowable number of treatments (following sessions \#29, \#36, and \#37). Based on our clinical observations, increased hopelessness following weeks of daily treatments without substantial improvement contributed to decompensation at the end of the course for three nonresponders. Increased impulsivity or substance use characterized adverse events for two veterans. Without a sham comparison condition, it is difficult to know whether the events were specifically related to rTMS.

We also observed significant rates of TMS-associated activation with $5 \mathrm{~Hz}$ TMS. We are not able to compare these rates to similar studies using $10 \mathrm{~Hz}$ TMS (e.g., O'Reardon et al., 2007, George et al., 2010, Levkovitz et al., 2015) as those studies did not include patients with comorbid PTSD. As noted above, the vast majority of these participants were taking stimulants or related compounds, and further research is needed to prospectively characterize and evaluate the relationship between TMS-related activation, psychiatric comorbidity, and CNS-stimulating drugs.

While $5 \mathrm{~Hz}$ rTMS as an adjunct to ongoing pharmacotherapy regimens is generally well tolerated (Philip et al., 2015), we observed that patients in this study taking concurrent stimulant medications or highly noradrenergic agents were still vulnerable to experiencing excessive activation (emergence or worsening of insomnia, irritability, anxiety, or agitation) with daily treatments. rTMS-emergent signs of activation can be detected early with focused daily clinical assessment and are easily managed in the majority of cases, but dose reduction or discontinuation of nonessential agents with stimulating CNS effects prior to starting a course of TMS may represent the best strategy for clinical practice. 
We found that $5 \mathrm{~Hz}$ rTMS to left PFC appeared to work well for reducing symptoms of both comorbid disorders rather than preferentially acting on either MDD or PTSD symptom domains. Our data revealed that baseline-to-endpoint change on PTSD and MDD scales were highly correlated throughout the study. These findings are consistent with our prior case-series observation that $5 \mathrm{~Hz}$ rTMS was helpful in PTSD (Philip et al., 2016), yet stands in contrast to results of Boggio et al. (2010) showing differential effects on mood versus anxiety symptoms during a 10-session course of $20 \mathrm{~Hz}$ rTMS based on laterality. We chose an "intermediate" frequency parameter, i.e., higher than $1 \mathrm{~Hz}$ but lower than $10 \mathrm{~Hz}$, which might have accounted for the broader benefits observed in our patient sample. More research is needed to elucidate the therapeutic mechanisms of rTMS delivered across differing pulse frequencies, and how underlying neurobiological changes translate to narrow or broad domains of clinical improvement.

Several factors were associated with nonresponse. Greater depressive symptom severity predicted worse response, consistent with previously reported predictors of nonresponse to antidepressant medication (Kilts et al., 2009) and to rTMS in a large naturalistic sample that likely included patients with diagnostic comorbidities (Carpenter et al., 2012). Additionally, a greater degree of PTSD avoidance symptoms at baseline predicted inferior outcomes. The type of stimulation we applied in this trial may have been insufficient or counterproductive for engaging the circuitry associated with avoidance symptoms. Behavior therapy specifically targeting avoidance in concert with rTMS may be required to see benefit in this domain.

\section{Strengths and limitations}

A significant strength of this study is the focus on a patient population that has been excluded from rTMS registration trials yet commonly presents in treatment settings. The majority of participants were fully disabled due to their symptoms. In addition to improvements in both MDD and PTSD symptoms domains, we found rTMS significantly reduced subjective stress levels, as measured by the DASS and PSS. There was limited racial and ethnic diversity in our sample due to the demographic characteristics of patients in our clinical practice settings. However, individuals with both early childhood trauma as well as adult onset (combat exposure) were included. Except as noted above, we required medications to be stable, which reduces but does not eliminate the possibility that observed positive effects were attributable at least in some part to ongoing psychotropic medications. Use of concurrent medications may be considered a limitation when evaluating the efficacy of rTMS in any sample, but we identified a pressing need for data describing naturalistic outcomes to inform everyday clinical practice. Furthermore, we allowed ongoing concurrent psychotherapy to continue on a stable schedule during the trial, so it is possible our results also incorporated additive effects or synergy between rTMS and psychotherapy. The lack of a sham comparator limits our ability to attribute findings to stimulation with the highest level of confidence. Due to sample size, our exploration of clinical predictors of response was limited to correlational analyses of baseline severity ratings on clinical measures. Future studies, using larger sample sizes, will need to evaluate the impact of other potentially informative candidate clinical variables (e.g., trauma type, childhood vs. adult onset, duration of illness, baseline medication status). Other limitations include modest sample 
size, limitations inherent to retrospective assessments of trauma and symptom onset, and lack of assessments to detect impact of the treatment on quality of life or functional status.

\section{Conclusions}

The current study demonstrates the feasibility, safety and potential efficacy of $5 \mathrm{~Hz}$ rTMS for individuals with comorbid PTSD and MDD. Though they were not represented in the regulatory trials, many patients with this common clinical comorbidity appear to benefit from rTMS therapy and tolerate it well as an adjunct to psychotropic medications when delivered with a $5 \mathrm{~Hz}$ protocol.

\section{Supplementary Material}

Refer to Web version on PubMed Central for supplementary material.

\section{DECLARATIONS OF INTEREST:}

This study was funded in part by an investigator-initiated grant from Neuronetics, Inc. to Butler Hospital (Drs. Carpenter and Philip). Drs. Carpenter, Tyrka and Price have received grant support from Janssen, Neosync, and Cervel Neurotech through clinical trials contracts with Butler Hospital. Neosync and Neuronetics have provided devices for research underway by several coauthors. Dr. Carpenter has received consulting income from Magstim Ltd. Dr. Philip has served as an unpaid scientific advisory board member for Neuronetics. All other authors report no biomedical financial interests or potential conflicts of interest.

\section{REFERENCES}

Alim TN, Feder A, Graves RE, Wang Y, Weaver J, Westphal M, et al., 2008 Trauma, resilience, and recovery in a high-risk African-American population. Am. J. Psychiatry 165(12), 1566-75. doi: 10.1176/appi.ajp.2008.07121939 [PubMed: 19015233]

Avery DH, Isenberg KE, Sampson SM, Janicak PG, Lisanby SH, Maixner DF, et al., 2008 Transcranial magnetic stimulation in the acute treatment of major depressive disorder: clinical response in an open-label extension trial. J Clin Psychiatry 69(3), 441-51. [PubMed: 18294022]

Berlim MT, Van den Eynde F, Daskalakis ZJ. 2013 Clinically meaningful efficacy and acceptability of low-frequency repetitive transcranial magnetic stimulation (rTMS) for treating primary major depression: a meta-analysis of randomized, double-blind and sham-controlled trials. Neuropsychopharmacology 38(4), 543-51. doi: 10.1038/npp.2012.237 [PubMed: 23249815]

Bernstein DP, Stein JA, Newcomb MD, Walker E, Pogge D, Ahluvalia T, et al., 2003 Development and validation of a brief screening version of the Childhood Trauma Questionnaire. Child Abuse Negl 27(2), 169-190. doi: 10.1016/S0145-2134(02)00541-0 [PubMed: 12615092]

Boggio PS, Rocha M, Oliveira MO, Fecteau S, Cohen RB, Campanha C, et al., 2010 Noninvasive brain stimulation with high-frequency and low-intensity repetitive transcranial magnetic stimulation treatment for posttraumatic stress disorder. J. Clin. Psychiatry 71(8), 992-9. doi: 10.4088/JCP. 08m04638blu [PubMed: 20051219]

Campbell DG, Felker BL, Liu CF, Yano EM, Kirchner JE, Chan D, et al., 2007 Prevalence of depression-PTSD comorbidity: implications for clinical practice guidelines and primary care-based interventions. J. Gen. Intern. Med 22(6), 711-8. doi: 10.1007/s11606-006-0101-4 [PubMed: 17503104]

Carpenter LL, Janicak PG, Aaronson ST, Boyadjis T, Brock DG, Cook IA, et al., 2012 Transcranial magnetic stimulation (TMS) for major depression: a multisite, naturalistic, observational study of acute treatment outcomes in clinical practice. Depress Anxiety 29(7), 587-96. doi: 10.1002/da. 21969 [PubMed: 22689344]

Clark C, Cole J, Winter C, Williams K, Grammer G. 2015 A Review of Transcranial Magnetic Stimulation as a Treatment for Post-Traumatic Stress Disorder. Curr. Psychiatry Rep 17(10), 83. doi: 10.1007/s11920-015-0621-x [PubMed: 26324821] 
Cohen S, Kamarck T, Mermelstein R. 1983 A global measure of perceived stress. J. Health. Soc. Behav 24(4), 385-96. [PubMed: 6668417]

Enticott PG, Fitzgibbon BM, Kennedy HA, Arnold SL, Elliot D, Peachey A, et al., 2014 A doubleblind, randomized trial of deep repetitive transcranial magnetic stimulation (rTMS) for autism spectrum disorder. Brain Stimul 7(2), 206-211. doi: 10.1016/j.brs.2013.10.004 [PubMed: 24280031]

Fitzgerald PB, McQueen S, Herring S, Hoy K, Segrave R, Kulkarni J, et al., 2009 A study of the effectiveness of high-frequency left prefrontal cortex transcranial magnetic stimulation in major depression in patients who have not responded to right-sided stimulation. Psychiatry Res169(1), 12-5. doi: 10.1016/j.psychres.2008.06.017 [PubMed: 19631391]

Flory JD, Yehuda R. 2015 Comorbidity between post-traumatic stress disorder and major depressive disorder: alternative explanations and treatment considerations. Dialogues Clin. Neurosci 17(2), 141-50. [PubMed: 26246789]

George MS, Lisanby SH, Avery D, McDonald WM, Durkalski V, Pavlicova M, et al., 2010 Daily left prefrontal transcranial magnetic stimulation therapy for major depressive disorder: a shamcontrolled randomized trial. Arch. Gen. Psychiatry 67(5), 507-16. doi: 10.1001/archgenpsychiatry. 2010.46 [PubMed: 20439832]

George MS, Nahas Z, Molloy M, Speer AM, Oliver NC, Li XB, et al., 2000 A controlled trial of daily left prefrontal cortex TMS for treating depression. Biol. Psychiatry 48(10), 962-70. [PubMed: 11082469]

Guy W 1976 Early Clinical Drug Evaluation Unit (ECDEU) assessment manual for psychopharmacology (Revised) National Institute of Mental Health, Bethesda.

Hoge CW, Castro CA, Messer SC, McGurk D, Cotting DI, Koffman RL. 2004 Combat duty in Iraq and Afghanistan, mental health problems, and barriers to care. N. Engl. J. Med 351(1), 13-22. doi: 10.1056/NEJMoa040603 [PubMed: 15229303]

Holtzheimer PE, Russo J, Zatzick D, Bundy C, Roy-Byrne PP. The impact of comorbid posttraumatic stress disorder on short-term clinical outcome in hospitalized patients with depression. Am J Psychiatry 2005;162(5):970-6. doi: 10.1176/appi.ajp.162.5.970 [PubMed: 15863800]

Karsen EF, Watts BV, Holtzheimer PE. 2014 Review of the effectiveness of transcranial magnetic stimulation for post-traumatic stress disorder. Brain Stimul 7(2), 151-7. doi: 10.1016/j.brs. 2013.10.006 [PubMed: 24486424]

Kessler RC, Berglund P, Demler O, Jin R, Merikangas KR, Walters EE. 2005 Lifetime prevalence and age-of-onset distributions of DSM-IV disorders in the National Comorbidity Survey Replication. Arch. Gen. Psychiatry 62(6), 593-602. doi: 10.1001/archpsyc.62.6.593 [PubMed: 15939837]

Kilts CD, Wade AG, Andersen HF, Schlaepfer TE. 2009 Baseline severity of depression predicts antidepressant drug response relative to escitalopram. Expert Opin Pharmacother 10(6), 927-36. doi: 10.1517/14656560902849258 [PubMed: 19317630]

Kroenke K, Spitzer RL, Williams JB. 2001 The PHQ-9: validity of a brief depression severity measure. J. Gen. Intern. Med 16(9), 606-13. doi: 10.1046/j.1525-1497.2001.016009606.x [PubMed: 11556941]

Lakens D 2013 Calculating and reporting effect sizes to facilitate cumulative science: a practical primer for t-tests and ANOVAs. Frontiers in Psychology 4(863). doi: 10.3389/fpsyg.2013.00863

Levkovitz Y, Isserles M, Padberg F, Lisanby SH, Bystritsky A, Xia G, et al., 2015 Efficacy and safety of deep transcranial magnetic stimulation for major depression: a prospective multicenter randomized controlled trial. World Psychiatry 14(1), 64-73. doi: 10.1002/wps.20199 [PubMed: 25655160]

Lisanby SH, Husain MM, Rosenquist PB, Maixner D, Gutierrez R, Krystal A, et al., 2009 Daily left prefrontal repetitive transcranial magnetic stimulation in the acute treatment of major depression: clinical predictors of outcome in a multisite, randomized controlled clinical trial. Neuropsychopharmacology 34(2), 522-34. doi: 10.1038/npp.2008.118 [PubMed: 18704101]

Lovibond SH, Lovibond PF. 1995 Manual for the Depression Anxiety \& Stress Scales, second ed. Psychology Foundation, Sydney. 
McClintock SM, Reti IM, Carpenter LL, McDonald WM, Dubin M, Taylor SF, et al., 2017 Consensus Recommendations for the Clinical Application of Repetitive Transcranial Magnetic Stimulation (rTMS) in the Treatment of Depression. J. Clin. Psychiatry (In Press) doi: 10.4088/JCP.16cs10905

Nahas Z, Kozel FA, Li X, Anderson B, George MS. 2003 Left prefrontal transcranial magnetic stimulation (TMS) treatment of depression in bipolar affective disorder: a pilot study of acute safety and efficacy. Bipolar Disord 5(1), 40-7. doi: 10.1034/j.1399-5618.2003.0001.x [PubMed: 12656937]

O'Reardon JP, Solvason HB, Janicak PG, Sampson S, Isenberg KE, Nahas Z, et al., 2007 Efficacy and safety of transcranial magnetic stimulation in the acute treatment of major depression: a multisite randomized controlled trial. Biol. Psychiatry 62(11), 1208-16. doi: 10.1016/j.biopsych. 2007.01.018 [PubMed: 17573044]

Philip NS, Carpenter SL, Ridout SJ, Sanchez G, Albright SE, Tyrka AR, et al., 2015 5Hz Repetitive transcranial magnetic stimulation to left prefrontal cortex for major depression. J. Affect. Disord 186, 13-7. doi: 10.1016/j.jad.2014.12.024 [PubMed: 26210705]

Philip NS, Ridout SJ, Albright SE, Sanchez G, Carpenter LL. 2016 5-Hz Transcranial Magnetic Stimulation for Comorbid Posttraumatic Stress Disorder and Major Depression. J. Trauma. Stress 29(1), 93-6. doi: 10.1002/jts.22065 [PubMed: 26748883]

Rosenberg PB, Mehndiratta RB, Mehndiratta YP, Wamer A, Rosse RB, Balish M. 2002 Repetitive transcranial magnetic stimulation treatment of comorbid posttraumatic stress disorder and major depression. J. Neuropsychiatry Clin. Neurosci 14(3), 270-6. doi: 10.1176/jnp.14.3.270 [PubMed: 12154150]

Rossini D, Lucca A, Magri L, Malaguti A, Smeraldi E, Colombo C, Zanardi R. 2010 A symptomspecific analysis of the effect of high-frequency left or low-frequency right transcranial magnetic stimulation over the dorsolateral prefrontal cortex in major depression. Neuropsychobiology 62(2), 91-7. doi: 10.1159/000315439 [PubMed: 20523080]

Rumi DO, Gattaz WF, Rigonatti SP, Rosa MA, Fregni F, Rosa MO, et al., 2005 Transcranial magnetic stimulation accelerates the antidepressant effect of amitriptyline in severe depression: a doubleblind placebo-controlled study. Biol. Psychiatry 57(2), 162-6. doi: 10.1016/j.biopsych. 2004.10.029 [PubMed: 15652875]

Rush AJ, Gullion CM, Basco MR, Jarrett RB, Trivedi MH. 1996 The Inventory of Depressive Symptomatology (IDS): psychometric properties. Psychol. Med 26(3), 477-86. doi: 10.1017/ S0033291700035558 [PubMed: 8733206]

Rush AJ, Trivedi MH, Wisniewski SR, Nierenberg AA, Stewart JW, Warden D, et al., 2006 Acute and longer-term outcomes in depressed outpatients requiring one or several treatment steps: a STAR*D report. Am. J. Psychiatry 163(11), 1905-17. doi: 10.1176/ajp.2006.163.11.1905 [PubMed: 17074942]

Rytwinski NK, Scur MD, Feeny NC, Youngstrom EA. 2013 The co-occurrence of major depressive disorder among individuals with posttraumatic stress disorder: a meta-analysis. J. Trauma. Stress 26(3), 299-309. doi: 10.1002/jts.21814 [PubMed: 23696449]

Su TP, Huang CC, Wei IH. 2005 Add-on rTMS for medication-resistant depression: a randomized, double-blind, sham-controlled trial in Chinese patients. J. Clin. Psychiatry 66(7), 930-7. [PubMed: 16013911]

Thomas JL, Wilk JE, Riviere LA, McGurk D, Castro CA, Hoge CW. 2010 Prevalence of mental health problems and functional impairment among active component and National Guard soldiers 3 and 12 months following combat in Iraq. Arch. Gen. Psychiatry 67(6), 614-23. doi: 10.1001/ archgenpsychiatry.2010.54 [PubMed: 20530011]

Trivedi MH, Rush AJ, Wisniewski SR, Nierenberg AA, Warden D, Ritz L, et al., 2006 Evaluation of outcomes with citalopram for depression using measurement-based care in STAR*D: implications for clinical practice. Am. J. Psychiatry 163(1), 28-40. doi: 10.1176/appi.ajp.163.1.28 [PubMed: 16390886]

Watts BV, Landon B, Groft A, Young-Xu Y. 2012 A sham controlled study of repetitive transcranial magnetic stimulation for posttraumatic stress disorder. Brain Stimul 5(1), 38-43. doi: 10.1016/ j.brs.2011.02.002 [PubMed: 22264669] 
Watts BV, Schnurr PP, Mayo L, Young-Xu Y, Weeks WB, Friedman MJ. 2013 Meta-analysis of the efficacy of treatments for posttraumatic stress disorder. J. Clin. Psychiatry 74(6), e541-50. doi: 10.4088/JCP.12r08225 [PubMed: 23842024]

Weathers FW, Blake DD, Schnurr PP, Kaloupek DG, Marx BP, Keane TM. 2013 The Life Events Checklist for DSM-5 (LEC-5). The National Center for PTSD https://www.ptsd.va.gov/ professional/assessment/te-measures/life_events_checklist.asp (accessed August 2017).

Weathers FW, L BT, Keave TM, Palmieri PA, Marx BP, Schnurr P. 2013 The PTSD Checklist for DSM-5 (PCL-5). National Center for PTSD https://www.ptsd.va.gov/professional/assessment/ adult-sr/ptsd-checklist.asp (accessed August 2017). 
Table 1.

Baseline demographic and clinical characteristics

\begin{tabular}{|c|c|}
\hline Characteristics & $\mathbf{N}=\mathbf{3 5}$ \\
\hline Study site, $\mathrm{n}(\%)$ & $\begin{array}{l}\text { BH: } 18(51.4 \%) \\
\text { VA: } 17(48.6 \%)\end{array}$ \\
\hline Age, mean (SD, range) & $51.6(10.3,27-69)$ \\
\hline Sex, $\mathrm{n}(\%)$ & $\begin{array}{l}\text { Male: } 21(60.0 \%) \\
\text { Female: } 14(40.0 \%)\end{array}$ \\
\hline Race, $\mathrm{n}(\%)$ & $\begin{array}{l}\text { White: } 33(94.3 \%) \\
\text { Black: } 1(2.9 \%) \\
\text { More than one race: } 1(2.9 \%)\end{array}$ \\
\hline Highest level of education, $\mathrm{n}(\%)$ & $\begin{array}{l}\text { Less than high school: } 1(2.9 \%) \\
\text { High school or equivalent: } 7(20.0 \%) \\
\text { Vocational degree or some college: } 15(42.9 \%) \\
\text { College or advanced degree: } 12(34.2 \%)\end{array}$ \\
\hline Employment status, n (\%) & $\begin{array}{l}\text { Full time: } 5(14.3 \%) \\
\text { Unemployed: } 3(8.6 \%) \\
\text { Disability: } 21(60.0 \%) \\
\text { Retired: } 6(17.1 \%)\end{array}$ \\
\hline Prior ECT & $1(2.9 \%)$ \\
\hline History of psychiatric hospitalization & $21(60.0 \%)$ \\
\hline History of psych partial hospitalization & $17(48.6 \%)$ \\
\hline \multicolumn{2}{|l|}{ Childhood Trauma Questionnaire (CTQ) ${ }^{*}$} \\
\hline Total score, mean (SD) & $71.6(18.5)$ \\
\hline \multicolumn{2}{|c|}{ CTQ Categories, n (\% meeting subscale score for at least "moderate" degree of trauma) } \\
\hline Emotional abuse & $21(60 \%)$ \\
\hline Physical abuse & $14(40 \%)$ \\
\hline Sexual abuse & $26(74.3 \%)$ \\
\hline Emotional neglect & $19(54.2 \%)$ \\
\hline Physical neglect & $20(57.1 \%)$ \\
\hline Any subtype of maltreatment, n (\%) & $29(82.9 \%)$ \\
\hline \multicolumn{2}{|l|}{ Life Events Checklist (LEC) ${ }^{* *}$} \\
\hline Natural disaster, n (\%) & $7(20.0 \%)$ \\
\hline Fire or explosion, $\mathrm{n}(\%)$ & $7(20.0 \%)$ \\
\hline Transportation accident, $\mathrm{n}(\%)$ & $13(37.1 \%)$ \\
\hline Serious accident, $\mathrm{n}(\%)$ & $10(28.6 \%)$ \\
\hline Exposure to toxic substance, $\mathrm{n}(\%)$ & $2(5.7 \%)$ \\
\hline Physical assault, n (\%) & $17(48.6 \%)$ \\
\hline Assault with a weapon, $\mathrm{n}(\%)$ & $9(25.7 \%)$ \\
\hline Sexual assault, n (\%) & $14(40.0 \%)$ \\
\hline Other unwanted sexual experience, n (\%) & $17(48.6 \%)$ \\
\hline Combat or war-zone exposure, n (\%) & $8(22.9 \%)$ \\
\hline Captivity, n (\%) & $1(2.9 \%)$ \\
\hline Life-threatening illness/injury, n (\%) & $11(31.4 \%)$ \\
\hline
\end{tabular}




\begin{tabular}{|l|l|}
\hline Characteristics & $\mathbf{N}=\mathbf{3 5}$ \\
\hline Severe human suffering, $\mathrm{n}(\%)$ & $11(31.4 \%)$ \\
\hline Sudden violent death, $\mathrm{n}(\%)$ & $8(22.9)$ \\
\hline Sudden accidental death, $\mathrm{n}(\%)$ & $7(20.0)$ \\
\hline Caused serious harm, $\mathrm{n}(\%)$ & $4(11.4)$ \\
\hline Other, $\mathrm{n}(\%)$ & $11(31.4)$ \\
\hline
\end{tabular}

*TQ not completed by one subject.

** LEC items endorsed as "Happened to me" or "Witnessed" 
Table 2.

\section{Outcome measures}

\begin{tabular}{|c|c|c|c|c|}
\hline Measure & Baseline & Post-treatment & $\mathbf{t}$ & $p$ \\
\hline \multicolumn{5}{|l|}{ Primary outcome measures } \\
\hline IDS-SR total score, mean (SD) & $47.8(11.9)$ & $30.9(18.9)$ & 6.6 & $<.001$ \\
\hline IDS-SR percent change, mean (SD), range & & $\begin{array}{l}37.6 \%(32.3), \\
-26.5 \%-86.0 \%\end{array}$ & & \\
\hline PCL-5 total score, mean (SD) & $52.2(13.1)$ & $34.0(21.6)$ & 5.6 & $<.001$ \\
\hline PCL-5 percent change, mean (SD), range & & $\begin{array}{l}35.5(38.0) \\
-50.0 \%-90.9 \%\end{array}$ & & \\
\hline \multicolumn{5}{|l|}{ Secondary outcome measures } \\
\hline PHQ-9 total score, mean (SD) & $18.4(5.0)$ & $10.7(7.9)$ & 7.3 & $<.001$ \\
\hline DASS total score, mean (SD) & $67.4(26.7)$ & $43.7(32.5)$ & 5.4 & $<.001$ \\
\hline DASS Depression subscale total score, mean (SD) & $27.6(11.1)$ & $18.6(14.6)$ & 4.2 & $<.001$ \\
\hline DASS Anxiety subscale total score, mean (SD) & $17.0(9.5)$ & $10.9(9.5)$ & 5.6 & $<.001$ \\
\hline DASS Stress subscale total score, mean (SD) & $22.8(9.8)$ & $14.1(11.6)$ & 5.2 & $<.001$ \\
\hline PSS total score, mean (SD) & $34.9(6.6)$ & $27.1(10.2)$ & 4.9 & $<.001$ \\
\hline CGI-S score, mean (SD) & $5.1(0.6)$ & $2.8(1.7)$ & 7.1 & $<.001$ \\
\hline PGI-S score, mean (SD) & $5.4(0.7)$ & $4.3(1.2)$ & 4.7 & .001 \\
\hline CGI-I score, n (\%) & & $\begin{array}{l}\text { Very much improved: } 12(34.3 \%) \\
\text { Much improved: } 4(11.4 \%) \\
\text { Minimally improved: } 4(11.4 \%) \\
\text { No change: } 3(8.6 \%) \\
\text { Minimally worse: } 1(2.9 \%) \\
\text { Much worse: } 0\end{array}$ & & \\
\hline \multicolumn{5}{|l|}{ Categorical outcomes } \\
\hline IDS-SR Response, n (\%) & & $15(42.9)$ & & \\
\hline IDS-SR Remission, n (\%) & & $12(34.3)$ & & \\
\hline PCL-5 Response, n (\%) & & $17(48.6)$ & & \\
\hline Met response criteria on both IDS-SR and PCL-5, n (\%) & & $14(40.0)$ & & \\
\hline
\end{tabular}

Key: IDS-SR, Inventory of Depressive Symptomatology; SD, standard deviation; PCL-5, PTSD Checklist for DSM5; PHQ-9, Patient Health Questionnaire 9; DASS, Depression Anxiety Stress Scale; PSS, Perceived Stress Scale; CGI-S, Clinician-rated Clinical Global Impression of Severity; PGI-S, Patient-rated Clinical Global Impressions of Severity; CGI-I, Clinician-rated Clinical Global Impressions of Improvement. 
Table 3.

Point biserial correlation coefficients between baseline clinical measures and meeting response status on both IDS-SR (depression) and PCL-5 (PTSD) scales

\begin{tabular}{|l|l|}
\hline Baseline Measure & $r_{p b}$ \\
\hline IDS-SR total score & $-.38^{*}$ \\
\hline PCL-5 total score & -.21 \\
\hline PCL-5 subscales & \\
\hline Cluster B Re-Experiencing total score & -.11 \\
\hline Cluster C Avoidance total score & $-.42^{*}$ \\
\hline Cluster D Negative Cognitions and Mood total score & -.16 \\
\hline Cluster E Arousal total score & -.08 \\
\hline PSS total score & -.31 \\
\hline DASS-Anxiety total score & -.25 \\
\hline DASS-Stress total score & -.15 \\
\hline$*$ & \\
\hline$p<.05$ &
\end{tabular}

